\title{
Experimental Study on Microwave Pyrolysis Characteristics of Microalgae Chlorella Vulgaris
}

\author{
Chun-xiang CHEN* \\ College of Electrical Engineering, Guangxi University \\ University Road 100, Xixiangtang District \\ College of Electrical Engineering, Guangxi University \\ Nanning City 530004, PR China. \\ e-mail: xiangxiang570@163.com \\ www.gxu.edu \\ Feng CHEN \\ College of Electrical Engineering, Guangxi University \\ University Road 100, Xixiangtang District \\ Nanning City 530004, PR China
}

\begin{abstract}
Pyrolysis characteristics of Chlorella vulgaris (C. vulgaris) (a kind of microalgae) are studied by microwave oven. The effects of different dosages of C. vulgaris, different contents of activated carbon and different microwave absorption on the microwave pyrolysis of $\mathrm{C}$. vulgaris are investigated through pyrolysis temperature versus time plots. The results indicate that with the increasing dosage of C.vulgaris, pyrolysis temperature tend to drop. With the increasing the dosage of activated carbon, pyrolysis temperature rises.
\end{abstract}

Keywords- Microalgae; Chlorella vulgaris; Microwavepyrolysis; Dosages.

\section{INTRODUCTION}

Microwaves are a form of electromagnetic radiation with frequencies ranging from $300 \mathrm{MHz}$ to $300 \mathrm{GHz}$, and wavelength is between one meter and one millimeter [1]. Microwave heating is volumetric heating caused by dielectric loss in the electromagnetic field. Therefore, microwave heating is also called no temperature gradient "body heat"[2]. Compared with the traditional heating way, it has characters of fast heating, heating uniformity, energy saving, high efficiency and easy to control, selective heating and without safety, etc.

Among bio-fuel, microalgae is considered as one of the most promising and renewable energy sources because of its wide distribution, high oil content and short growth cycle, etc[3]. it is good to use microalgae toproduce bio-fuel[4].

This paper studies the temperature characteristics of $\mathrm{C}$. vulgaris by microwave oven. The influence factors, the dosage of $\mathrm{C}$. vulgaris, the content of activated carbon and different kinds of microwave absorption, are studied. The purpose is to obtain the rules of $\mathrm{C}$. vulgaris during microwave pyrolysis. The rules of three influence factors on microwave pyrolysis temperature are also indicated.

\author{
Zheng CHENG \\ College of Electrical Engineering, Guangxi University \\ University Road 100, Xixiangtang District \\ Nanning City 530004, PR China.
}

\section{EXPERIMENTAL FACILITIES AND METHODOLOGY}

The experimental device of microwave system was shown in Fig. 1 and the experiment device is mainly composed of microwave occurrence and control system, temperature measuring system, material reaction system, pyrolysis products collection system and data acquisition system. In this study, activated carbon was used as microwave absorption. The type of the chosen activated carbon was super fine powder, so put it directly sieved with a mesh size of 80 . Then activated carbon and C. vulgaris (dry basis) could be uniform mixing. Different contents of activated carbon with $30 \mathrm{~g}$ microalgae (dry basis) evenly and configure different contents of activated carbon $(0 \%, 3 \%$, $5 \%, 7 \%, 10 \%, 20 \%$ ) for the preparation. The power of microwave oven is $1500 \mathrm{w}$.

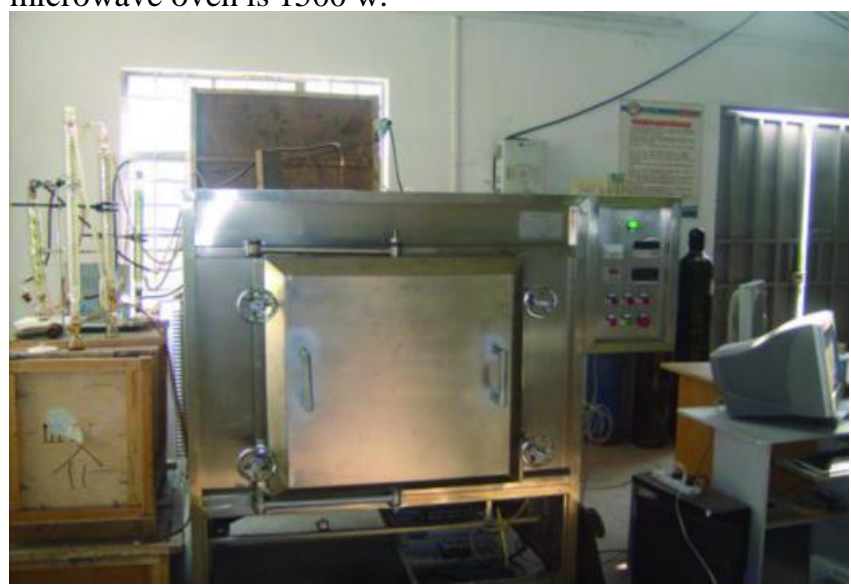

Fig. 1. The experimental system diagram of microwave pyrolysis device 


\section{RESULTS AND DISCUSSION}

A. Effect of Different Contents of Activated Carbon on the Microwave Pyrolysis of C.vulgaris

Heating curves of C. vulgaris with different activated carbon contents during microwave pyrolysis is shown in Fig.2.

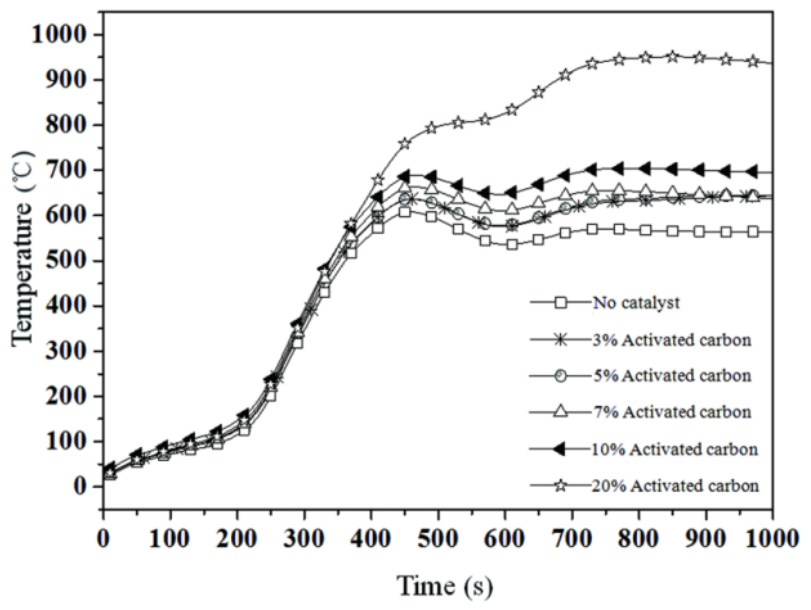

Fig.2. Heating curves of C. vulgaris with different activated carbon contents

As can be seen from Fig.2, except the sample which is added $20 \%$ activated carbon, pyrolysis performance of the other four curves turns out to be similar, there are 5 stages during pyrolysis process. The curve of the sample which is added 20\% activated carbon illustrates four stages. When compared with pyrolysis curves of $0 \%-10 \%$ activated carbon content, before the pyrolysis time is about $375 \mathrm{~s}$, the pyrolysis curve of $20 \%$ activated carbon content appears to be extremely similar to that of $0 \% \sim 10 \%$; After 375 s, pyrolysis temperature keeps on rising until reaching the pyrolytic stability stage and there is not a temperature-fall stage in the whole process. Compared with the sample without catalyst, only after the pyrolysis time is about 350s, pyrolysis temperature of different pyrolysis curves rises with the increase of activated carbon content. That is because the longer the activated carbon stays in microwave field, the larger its porosity as well as specific surface area is, so that they will favour C. vulgaris's pyrolysis more. The sample added 5\% activated carbon takes the least time to reach the balance of pyrolysis process.

\section{B. Effect of Different Dosages of C.vulgaris on Its Microwave Pyrolysis}

Heating curves of C.vulgaris with different dosages $(15,20,25,30,35$ and $40 \mathrm{~g})$ during microwave pyrolysis is shown in Fig.3.

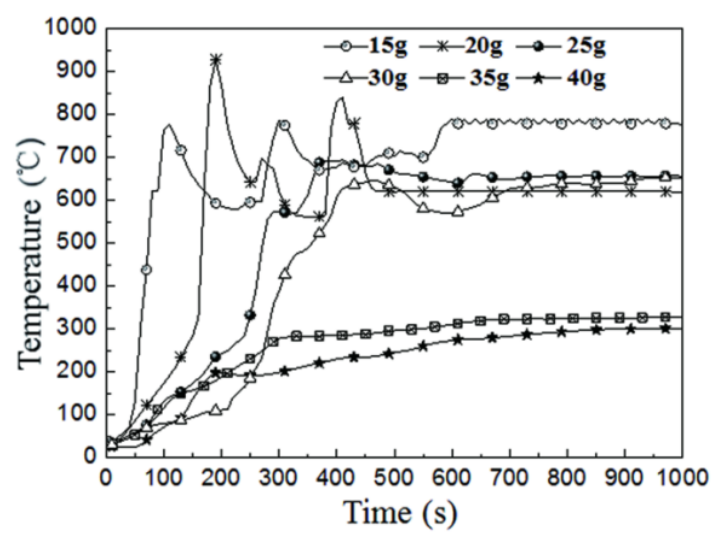

Fig. 3. Heating curves of C.vulgaris with different dosages during microwave pyrolysis

As can be seen from Fig.3, with increasing the dosage of C. vulgaris, pyrolysis temperature tend to drop. When the dosage of C. vulgaris increases from 15 to $30 \mathrm{~g}$, pyrolysis temperature rises firstly and then drops, finally it tends to be stable; however, when the dosage of material reaches $35 \mathrm{~g}$, pyrolysis temperature curves is gentle and its temperature rises slowly to plateau. When the dosage of material increases from 15 to $45 \mathrm{~g}$, the highest pyrolysis temperature of C. vulgaris increases from 301.86 to $927.84^{\circ} \mathrm{C}$. And when the dosage of material is $15,20,25,30,35$ and $40 \mathrm{~g}$, the final pyrolysis temperature is $783,620,655,650,320$ and $300^{\circ} \mathrm{C}$, and the pyrolysis time is $600,475,735,780,965$ and 920 s, respectively. It is obvious that as the dosage of material increases, the final pyrolysis temperature is lower, the pyrolysis time is longer. That is because microwave energy is attenuated when it comes into the inside of sample, and as the dosage of material increases, the thickness of material will be thicker, the internal areas which are far away from the surface of material[4]. Therefore, the dosage of material has an effect on pyrolysis process obviously.

\section{CONCLUSION}

In this paper, the pyrolysis characteristics of C.vulgaris by microwave oven were studied. The following conclusions could be drawn from this work.

(1) With increasing the dosage of activated carbon, pyrolysis temperature rises.

(2) When the dosage of C.vulgaris increases, pyrolysis temperature tends to drop.

\section{ACKNOWLEDGMENT}

This work is supported by Guangxi Natural Science Foundation (2014GXNSFBA118252), Guangxi Scientific Research and Technology Development Project (GuiKegong 1598008-17) and University scientific research key project of Guangxi Zhuang Autonomous Region Education Department (ZD2014008). 


\section{REFERENCES}

[1] J.N. He, Q. Xu, W.G. Su, J.S. Ye. Study on application of microwave drying technology [J].Ying Yong Jiao Liu, 2006. 29(137): 55-56. (In Chinese).

[2] X.Q. Zhao, Z.L. Song, T. Wang, L.Z. Li, C.Y. MA. Progress of pyrolysis using microwave heating technique [J]. Chemical Industry and Engineering Progress, 2008. 27(12):1873-1881. (In Chinese).
[3] Luyao Zhang and Xuejing Li. 2009. The present situation and application prospect of biofuels prepared by microalage. Lubes \&Fuels. 19(5-6): 15-18.

[4] ZHAO Xiqiang, SONG Zhanlong, WANG Tao, LI Longzhi, MA Chunyuan. Progress of pyrolysis using microwave heating technique[J]. CHEMICAL INDUSTRY AND ENGINEERING PROGRESS, 2008, 27(12): 1873-1881. (In Chinese). 